\title{
ON A PARAMETRIZATION OF A CERTAIN ALGEBRAIC CURVE OF GENUS 2
}

\author{
ALEXANDER I. APTEKAREV, DMITRIY N. TOULYAKOV, AND MAXIM L. YATTSELEV
}

AвSTRACT. A parametrization of a certain algebraic curve of genus 2, given by a cubic equation, is obtained. This curve appears in the study of Hermite-Padé approximants for a pair of functions with overlapping branch points on the real line. The suggested method of parametrization can be applied to other cubic curves as well.

\section{INTRODUCTION}

In this note we find an explicit parametrization of the form $(w(t), z(t))$ of an algebraic curve in $\mathbb{C}^{2}=\mathbb{C}_{w} \times C_{z}$ given by

$$
w^{3}-3 \mathrm{~A}(z) w-2 \mathrm{~B}(z)=0,
$$

where

$$
\mathrm{A}(z):=\frac{1}{z^{2}-1}, \quad \mathrm{~B}(z):=\frac{z}{\left(z^{2}-\mathrm{a}^{2}\right)\left(z^{2}-1\right)}, \quad \mathrm{a} \in(0,1) .
$$

Methods of deriving explicit uniformizations (or more generally, parametrizations) of algebraic curves are of considerable independent interest as they simplify analysis of many objects associated with the curve, for example, of abelian integrals. Our own interest in the subject stems from Nuttall's conjecture on asymptotics of Hermite-Padé approximants to analytic vector-valued functions with branch points, see [6]. According to the conjecture, the asymptotic distribution of the poles of the approximants for functions of the form

$$
f_{j}(z):=\log \left(\frac{z-a_{j}}{z-b_{j}}\right), \quad j=1,2,
$$

is described with the help of the algebraic function (1), see [2] for details, for which

$$
A(z):=\frac{P_{2}(z)}{\Pi_{4}(z)}, \quad B(z):=\frac{P_{1}(z)}{\Pi_{4}(z)}, \quad \Pi_{4}(z):=\prod_{j=1}^{2}\left(z-a_{j}\right)\left(z-b_{j}\right),
$$

and the unknown coefficients of $\mathrm{P}_{\mathrm{k}}(z)=z^{\mathrm{k}}+\cdots, \mathrm{k}=1,2$, are chosen so that the periods of the abelian integral $\int w \mathrm{~d} z$ are purely imaginary, that is,

$$
\operatorname{Re} \oint_{\Gamma} w \mathrm{~d} z=0
$$

where $\Gamma$ is any cycle on the Riemann surface $\mathfrak{R}$ of (1) and (4). When the genus of $\mathfrak{R}$ is 1 or 2 , explicit elliptic and hyperelliptic uniformization were constructed in [4]. When genus is 0 , a rational uniformization were found in [ 1$]$.

The set up (1)-(2) is motived by the question of convergence of Hermite-Padé approximants for a pair of functions (3) with

$$
a_{1}=-1, \quad b_{1}=a, \quad a_{2}=-a, \quad b_{2}=1 ; \quad a \in(0,1 / \sqrt{2}) .
$$

Key words and phrases. Riemann surfaces, uniformization, parametrization of complex algebraic curves, abelian integrals, HermitePadé approximants.

The research of the first and second authors was supported by a grant from Russian Science Foundation, project RScF-14-21-00025.

This is the author's manuscript of the article published in final edited form as: 
This problem was investigated by Herbert Stahl $[7,3]$. However, a complete detailed solution was obtained only recently [5]. The fact that the only non-trivial period of the abelian integral (1)-(2) has a positive real part, i.e.,

$$
\exists \gamma \subset \mathfrak{R}: \quad \operatorname{Re} \oint_{\gamma} w \mathrm{~d} z>0, \quad a \in(0,1 / \sqrt{2}),
$$

was an essential ingredient for proofs in [5]. In what follows, we derive a parametrization of (1)-(2) and show how (5) follows from it.

\section{MAIN RESULTS}

The following theorem takes place.

Theorem 1. The curve (1)-(2) can be parametrized by

$$
\left\{\begin{array}{l}
w=-\mathrm{iC}\left(\varepsilon t+\frac{1}{\varepsilon t}\right), \quad \varepsilon^{3}=1, \quad C=\frac{\tilde{\tilde{a}}+\mathrm{it}^{3} \tilde{\mathrm{a}}}{\left(1-\mathrm{a}^{2}\right)\left(1+\mathrm{t}^{6}\right)^{\prime}}, \\
z=\frac{\tilde{\mathrm{a}}+\mathrm{it}^{3} \tilde{\tilde{\mathrm{a}}}}{1-\mathrm{t}^{6}},
\end{array}\right.
$$

where

$$
\tilde{a}:=\sqrt{a^{2}-\left(1-a^{2}\right) t^{6}}, \quad \tilde{a}:=\sqrt{\left(1-a^{2}\right)-a^{2} t^{6}} .
$$

In other words, Theorem 1 establishes a mapping of the Riemann surface $\mathfrak{H}$ of the vector function $(\tilde{\mathbf{a}}, \tilde{\tilde{a}})$ onto the Riemann Surface $\mathfrak{R}$ of the function (1)-(2). The surface $\mathfrak{H}$ can be realized as a four-sheeted surface of $H=a^{2} \tilde{a}-\left(1-a^{2}\right) \tilde{a}$ over $\mathbb{C}_{t}$. The roots in (7) need to be understood as analytic continuations of the principal branches.

Before we continue with the results, let us fix the global sheet structure of the algebraic function (1)-(2) (i.e., the realization of $\mathfrak{R}=\left\{\mathfrak{R}_{j}\right\}_{j=0}^{2}$ over $\mathbb{C}_{z}$ ). By letting $z \rightarrow \infty$, we can distinguish the branches

$$
w_{0}(z)=\frac{2}{z}+\cdots, \quad w_{i}(z)=-\frac{1}{z}+\cdots, \quad i=1,2 .
$$

The function $w=\left\{w_{j}(z)\right\}_{j=0}^{2}$ has 6 branch points: $z= \pm 1$ are branch points of the second order ( 2 branches of $w$ coincide there), $z= \pm a$ have order 3 , and $z= \pm i d, d=a^{2} / \sqrt{1-2 a^{2}}$ have order 2. In particular, $\mathfrak{R}$ has genus 2 .

Further analysis of (1)-(2) yields that the branches of $w$ fixed at $\infty$ posses holomorphic continuations into the following domains

$$
\left\{\begin{array}{l}
w_{0} \in \mathrm{H}(\overline{\mathbb{C}} \backslash[-1,1]), \\
w_{1} \in \mathrm{H}(\overline{\mathbb{C}} \backslash\{[-\mathrm{a}, \mathrm{a}] \cup[+\mathrm{id},+\mathrm{i} \infty] \cup[-\mathrm{id},-\mathrm{i} \infty]\}), \\
w_{2} \in \mathrm{H}(\overline{\mathbb{C}} \backslash\{[-1,-\mathrm{a}] \cup[\mathrm{a}, 1] \cup[+\mathrm{id},+\mathrm{i} \infty] \cup[-\mathrm{id},-\mathrm{i} \infty]\}) .
\end{array}\right.
$$

The cuts of the sheet $\Re_{j}$ are defined as the projection of the boundary of the domain of holomorphy of the corresponding branch $w_{j}$.

Let $t \in\left(0, t_{-}\right), t_{-}:=\left(a^{2} /\left(1-a^{2}\right)\right)^{1 / 6}$. Then the expressions under the square roots in (7) are positive and the parametrization (6) defines an analytic arc $\tilde{\gamma}:=z(t)$ in $\mathbb{C}$ connecting the points $z(0)=a$ and $z\left(t_{-}\right)=i a / \sqrt{1-2 a^{2}}>i d$. Let us define a closed contour $\gamma$ on $\mathfrak{R}$ in the following way. The contour starts at the triple branch point above $a$ and continues in $\Re_{1}$ along $\tilde{\gamma}$ until the preimage of $z\left(t_{-}\right)$, then it proceeds along the preimage of the imaginary axis until the branch point above id, where it crosses to $\mathfrak{R}_{2}$ and goes back to initial point along the path with the same projection as the one on $\mathfrak{R}_{1}$.

Corollary 1. The conclusion of (5) holds for the just constructed cycle $\gamma$.

Indeed, notice that $\operatorname{Re}_{1}(z)=\operatorname{Rew}_{2}(z), z \in[i d,+i \infty]$, and therefore

$$
\operatorname{Re} \oint_{\gamma} w \mathrm{~d} z=\int_{\tilde{\gamma}}\left(w_{1}(z)-w_{2}(z)\right) \mathrm{d} z=\int_{0}^{t_{-}}\left(w_{1}-w_{2}\right) \frac{\mathrm{d} z}{\mathrm{dt}} \mathrm{dt} .
$$


Let us point out that the choice of the branch (8) in parametrization (6) is done via fixing the value of $\varepsilon$, where $w_{1}$ corresponds to $\varepsilon=(\mathrm{i} \sqrt{3}-1) / 2$ and $w_{2}$ corresponds to $\varepsilon=$ $(-\mathrm{i} \sqrt{3}-1) / 2$. Therefore,

$$
w_{1}-w_{2}=\sqrt{3} C\left(t-\frac{1}{t}\right) .
$$

After simplification, we deduce from (6) that

$$
\frac{d z}{d t}=\frac{3 t^{5}\left(3 a^{2}-1+\left(a^{2}-1\right) t^{6}\right)}{\tilde{a}\left(t^{6}-1\right)^{2}}-i \frac{3 t^{2}\left(a^{2}-1+\left(3 a^{2}-1\right) t^{6}\right)}{\tilde{\tilde{a}}\left(t^{6}-1\right)^{2}} .
$$

Hence,

$$
\left(w_{1}-w_{2}\right) \frac{\mathrm{d} z}{\mathrm{dt}}=\frac{3 \sqrt{3}\left(1-2 \mathrm{a}^{2}\right)}{\tilde{\mathrm{a}} \tilde{\tilde{a}} \mathrm{t}^{2}\left(\mathrm{t}^{4}+\mathrm{t}^{2}+1\right)}-\mathrm{i} \frac{3 \sqrt{3} \mathrm{t}}{\mathrm{t}^{4}+\mathrm{t}^{2}+1},
$$

which proves the corollary.

\section{PROOF OF THEOREM I}

The approach we use to find the parametrization of (I) is rather general. It is based on the same ideas as Cardano's formulae. We represent $w=u+v$ and look for functions $u, v$ so that the branches $w_{j}$ are given by

$$
w_{j}=\varepsilon^{j} u+\varepsilon^{-j} v, \quad j=0,1,2, \quad \varepsilon^{3}=1 .
$$

The Vieta's relations for (I) yield that

$$
\left\{\begin{array}{l}
u^{3}+v^{3}=2 B(z) \\
u v=A(z)
\end{array} \Rightarrow\left(\frac{u^{3}-v^{3}}{u^{3}+v^{3}}\right)^{2}=\frac{B^{2}-A^{3}}{B^{2}} .\right.
$$

Choosing the parameter $\mathrm{t}$ as

$$
\mathrm{t}^{2}=\frac{\mathrm{u}}{v} \Rightarrow\left(\frac{\mathrm{t}^{6}-1}{\mathrm{t}^{6}+1}\right)^{2}=\frac{\mathrm{B}^{2}-\mathrm{A}^{3}}{\mathrm{~B}^{2}}=\frac{\left(1-2 \mathrm{a}^{2}\right) z^{2}+\mathrm{a}^{4}}{z^{2}\left(1-z^{2}\right)},
$$

which gives the dependence of $z$ on $t$. To find $w(t)$, let us write

$$
w=u\left(1+\frac{v}{u}\right), \quad u\left(\frac{\left(\frac{u}{v}\right)^{3}+1}{\left(\frac{u}{v}\right)^{2}}\right)=\frac{u^{3}+v^{3}}{u v}=\frac{2 B}{A} .
$$

Denoting $T_{ \pm}:=t^{6} \pm 1$, we can rewrite (9) as

which yields

$$
z^{4}-z^{2}\left(\frac{\mathrm{T}_{-}^{2}-\left(1-2 \mathrm{a}^{2}\right) \mathrm{T}_{+}^{2}}{\mathrm{~T}_{-}^{2}}\right)+\mathrm{a}^{4} \frac{\mathrm{T}_{+}^{2} \mathrm{~T}_{-}^{2}}{\mathrm{~T}_{-}^{4}}=0
$$

$$
z=\frac{1}{2 T_{-}}\left(R_{+} \pm R_{-}\right), \quad R_{ \pm}:=\sqrt{T_{-}^{2}-\left(1-2 a^{2}\right) T_{+}^{2} \pm 2 a^{2} T_{+} T_{-}} .
$$

After some algebra, we have

$$
R_{+}=2 t^{3} i \sqrt{\left(1-a^{2}\right)-a^{2} t^{6}}, \quad R_{-}=2 \sqrt{a^{2}-\left(1-a^{2}\right) t^{6}},
$$

which, with the notation from $(7)$, gives us the parametrization $z(t)$ in (6). Finally, by plugging (2) into the right-hand side of (10), we get

$$
w=\left(1+t^{-2}\right)\left(\frac{t^{4}}{t^{6}+1}\right) \frac{z^{2}-a^{2}}{2 z}
$$

Using the parametrization $z(t)$ in the above expression and simplifying the result gives us the parametrization $w(t)$ in (6). 


\section{REFERENCES}

[1] A.I. Aptekarev, V.A. Kalyagin, V.G. Lysov, and D.N. Toulyakov. Equilibrium of vector potentials and uniformization of the algebraic curves of genus O. J. Comput. Appl. Math., 233(602-616), 2009. I

[2] A.I. Aptekarev, A. Kuijlaars, and W. Van Assche. Asymptotics of Hermite-Padé rational approximants for two analytic functions with separated pairs of branch points (case of genus o). Int. Math. Res. Pap., 2008. I

[3] A.I. Aptekarev and H. Stahl. Asymptotics of Hermite-Padé polynomials. In A.A. Gonchar and E.B. Saff, editors, Progress in Approximation Theory, pages 127-167. Springer-Verlag, 1992. 2

[4] A.I. Aptekarev, D.N. Toulyakov, and W. Van Assche. Hyperelliptic uniformization of algebraic curves of the third order. J. of Comput. and Appl. Math., 2015. 1

[5] A.I. Aptekarev, W. Van Assche, and M. Yattselev. Hermite-Padé approximants for a pair of Cauchy transforms with overlapping symmetric supports. Submitted for publication. http://arxiv.org/abs/1505.03993. 2

[6] J. Nuttall. Asymptotics of diagonal Hermite-Padé polynomials. J. Approx. Theory, 42(4):299-386, 1984. I

[7] H. Stahl. Asymptotics of Hermite-Padé polynomials and related convergence results - a summary of results. In Nonlinear numerical methods and rational approximation, volume 43 of Math. Appl., pages 23-53. Reidel, Dordrecht, 1987.2

Keldysh Institute of Applied Mathematics, Russian Academy of Science, Moscow, 125047, Russia E-mail address: aptekaa@keldysh.ru

Keldysh Institute of Applied Mathematics, Russian Academy of Science, Moscow, 125047, Russia E-mail address: dntulyakov@gmail.com

Department of Mathematical Sciences, Indiana University-Purdue University Indianapolis, 402 North BLACKFORD STREET, INDIANAPOLIS, IN 46202

E-mail address: maxyatts@iupui.edu 